$62^{\text {ème }}$ Congrès de la SFCO, 03002 (2014)

DOI: $10.1051 /$ sfco/20146203002

(C) Owned by the authors, published by EDP Sciences, 2014

\title{
Aspects épidémiologiques et thérapeutiques des fractures mandibulaires au centre hospitalier national de Nouakchott.
}

\section{Veten $\mathbf{M}^{1}$, Tamba $\mathbf{B}^{2}$, DiaTine $\mathrm{S}^{2}$, Tichitty $\mathrm{A}^{\mathbf{1}}$, Moustapha $\mathbf{A}^{1}$}

1 Service de stomatologie - Centre Hospitalier National de Nouakchott, MAURITANIE

2 Service d'odontostomatologie - Hôpital Général de Grand Yoff, SÉNÉGAL

\section{Introduction}

L'épidémiologie et la prise en charge des fractures mandibulaires varient d'un pays à un autre, le but de ce travail était d'exposer les particularités de cette pathologie au centre hospitalier national de Nouakchott qui est la seule structure hospitalière en Mauritanie à prendre en charge ce genre de pathologie.

\section{Matériel et méthode}

II s'agit d'une étude rétrospective, menée de 2010 à 2012, qui a permis de répertorier 79 patients victimes de fracture mandibulaire et pris en charge au centre hospitalier national de Nouakchott. Une analyse épidémiologique, clinique, radiologique et thérapeutique y a été réalisée.

\section{Résultats}

Les patients étaient des hommes jeunes d'âge moyen de 29 ans. Les causes les plus fréquentes étaient les accidents de la voie publique (86\%), suivi par les accidents liées au sport et au jeux (10\%). Le corps de la mandibule était la localisation la plus fréquente (46\%),suivie par la région symphysaire (31\%). Le blocage intermaxillaire (BIM) a été le traitement le plus fréquent $(80 \%)$ suivi par L'ostéosynthèse par les mini plaques (13\%). Les troubles de l'articulé dentaire et les pseudarthroses ont été les séquelles les plus fréquentes.

\section{Discussion}

Les données épidémiologiques de cette étude correspondent globalement à celles retrouvées dans les pays de la région. L'analyse de telles données pourrait permettre de mieux organiser la prise en charge des traumatismes maxillo-faciaux en Mauritanie, Les choix thérapeutique dépendent du type de fracture mais également du niveau socioéconomique du patient.

VETEN Mohammed drveten-ucad@hotmail.fr

This is an Open Access article distributed under the terms of the Creative Commons Attribution License 4.0, which permits unrestricted use, distribution, and reproduction in any medium, provided the original work is properly cited. 